

\section{USO DE HERRAMIENTAS EN LÍNEA PARA EL APRENDIZAJE}

\section{Use of online tools for learning}

\section{BRENDA CECILIA PADILLA RODRÍGUEZ FRANCISCO JAVIER ROCHA ESTRADA}

\section{RESUMEN}

Las tecnologías de la información y comunicación han cambiado significativamente el ámbito educativo, los profesores han identificado su potencial y cada vez son más utilizadas en el salón de clases. Nuestro país ha tenido un lento desarrollo en relación a otros, pero los jóvenes cada vez utilizan más herramientas tecnológicas para su aprendizaje y, al conocer las herramientas tecnológicas que utilizan para su aprendizaje, se puede facilitar el proceso de enseñanza. Se utilizó un instrumento con cinco preguntas de opción múltiple y una pregunta abierta para conocer cuáles herramientas son más utilizadas por los estudiantes. Se calcularon porcentajes de las preguntas cerradas y se codificaron las preguntas abiertas para encontrar patrones. Los resultados muestran que los grupos colaborativos y los portales de videos son las herramientas más utilizadas por los jóvenes. Este conocimiento puede permitir a los maestros incluirlas en sus cursos y facilitar el proceso de enseñanza-aprendizaje de los estudiantes.

PALABRAS CLAVE: medios, tecnología, educación, enseñanza, aprendizaje, digital.

\section{ABSTRACT}

Information and communication technologies have significantly changed the educational environment; teachers have identified their potential and are increasingly used in the classroom. Our country has had a slow development in relation to others, but young people increasingly use more technological tools for their learning and, knowing the technological tools they use for their learning, can facilitate the teaching process. An instrument with five multiple choice questions was used and an open question to know which tools students use for their learning. Percentages of the closed questions were calculated and the open questions were coded to find patterns. The results show that collaborative groups and video portals are the tools most used by young people. This knowledge can allow teachers to include them in their courses and facilitate the teaching-learning process for the students.

KEYWORDS: media, technology, education, teaching, learning, digital. 
a educación en línea de México

ha tenido un lento desarrollo a

comparación de otros países, no obstante durante los últimos años se ha incrementado la prevalencia de las tecnologías de la información, principalmente entre los jóvenes. El presente estudio tiene como objetivo conocer las herramientas en línea que disponen los estudiantes universitarios y cuáles son las más relevantes para su aprendizaje.

Las tecnologías de la información y la comunicación cambiaron el proceso de enseñanza-aprendizaje. Su uso facilita el intercambio de información, rompe los límites espacio-temporales de la educación tradicional, promueve el rol activo de los estudiantes y brinda acceso a materiales de apoyo o enriquecimiento (Ferro, Martínez \& Otero, 2009). Un entorno interconectado permite el desarrollo de competencias digitales y favorece el aprendizaje colaborativo a través de las comunidades educativas (Prieto, 2013).

Profesores han reportado que la tecnología cuenta con potencial para mejorar la calidad del aprendizaje, fomenta el interés de los estudiantes en sus actividades, y ayuda a mejorar la cooperación, comunicación, compromiso, análisis, síntesis y evaluación de la información (Navaridas, Santiago \& Tourón, 2013).

En México, la educación en línea ha tenido un lento desarrollo a comparación de otros países (Cookson \& Domínguez, 2015). Por ejemplo, la velocidad promedio del internet es de 7.2 megabits por segundo, mientras que Estados Unidos tiene 16.3 mbps y Corea del Sur 26.3 mbps
(Akamai, 2016). A pesar de este desfase, durante los últimos años se ha incrementado la prevalencia de las tecnologías de la información, principalmente entre los jóvenes (Herrera \& Batista, 2009).

De acuerdo con la Encuesta Nacional sobre Disponibilidad y Uso de Tecnologías de la Información en Hogares (Instituto Nacional de Estadística, Geografía e Informática [INEGI], 2017), el 57.4\% de la población mexicana (62.4 millones de personas) usa internet. 9 de cada 10 jóvenes que cuentan con estudios de nivel superior lo utilizan habitualmente. Las actividades más comunes en línea se relacionan a la búsqueda de información, la comunicación, el acceso a contenidos audiovisuales, la participación en redes sociales y el apoyo a la educación, en este orden.

Existen diferentes herramientas tecnológicas que pueden usarse con fines de aprendizaje. Estas incluyen:

1. Servicios de Almacenamiento en la Nube: que permiten guardar archivos y acceder a ellos desde cualquier lugar con una conexión a internet (Morales, 2014).

2. Portales de Videos: que representan la herramienta audiovisual más utilizada por estudiantes (Cázares \& Urbiola, 2015).
3. Grupos Colaborativos: en los que frecuentemente se comparte información, recursos y opiniones (Humanante \& Ramos et al., 2015).

4. Bases de Datos Académicas: las cuales no siempre son conocidas por docentes (Infante et al., 2014).

5. Videoconferencias: que permiten una comunicación bidireccional y en tiempo real sin la necesidad de que los participantes se encuentren en un lugar específico (Álvarez et al., 2013).

El presente estudio tiene como objetivo conocer las herramientas en línea que disponen los estudiantes universitarios y cuáles son las más relevantes para su aprendizaje.

\section{METODOLOGÍA}

Se utilizó un diseño descriptivo, no experimental y transversal. La muestra fue seleccionada por conveniencia y contó con 100 estudiantes del primer semestre de la Facultad de Psicología de la UANL.

Se les invitó a participar de manera presencial yse les informó el propósito del estudio. Su participación fue libre, voluntaria y anónima. Los alumnos interesados contestaron una encuesta en línea.

\section{Las actividades más comunes en} línea se relacionan a la búsqueda de información, la comunicación, el acceso a contenidos audiovisuales, la participación en redes sociales y el apoyo a la educación, en este orden. 
El instrumento contenía cinco preguntas cerradas sobre el uso de herramientas en línea relacionadas a:

1. Almacenamiento en la nube

2. Portales de videos

3. Grupos colaborativos

4. Bases de datos académicas

5. Videoconferencias

Asimismo, se incluyó una pregunta abierta sobre otras herramientas utilizadas con fines de aprendizaje. Para analizar los resultados se calcularon los porcentajes de las respuestas, se realizó un análisis temático de las respuestas abiertas para identificar los patrones y las tendencias.

\section{RESULTADOS Y DISCUSIÓN}

La mayoría de los estudiantes reportaron estar familiarizados con herramientas en línea de los diferentes tipos (Herrera \& Batista, 2009; Tabla 1). Los grupos colaborativos en línea fueron los más utilizados con fines de aprendizaje. Esto empata con las estadísticas nacionales que resaltan el uso del internet con fines de comunicación y socialización (INEGI,
2016) y reportes previos (eg, Humanante \& Ramos et al., 2015).

El 24\% de los participantes mencionaron desconocer bases de datos académicas. Este tipo de reportes no es exclusivo de los estudiantes sino que también atañe a profesores universitarios (Infante et al., 2014). Los portales de videos son ampliamente utilizados, quizá por su frecuente difusión en redes sociales (Álvarez et al., 2013). Sin embargo, las videoconferencias no cuentan con la misma popularidad. Esto pudiera ser por el limitado avance de la educación a distancia en México (Cookson \& Domínguez, 2015) ya que requieren un mayor ancho de banda (Akamai, 2016).

En la pregunta abierta sobre otras herramientas utilizadas con fines de aprendizaje el $69 \%$ de los estudiantes no reportó ninguna otra. El resto proporcionó respuestas variadas, incluyendo:

1. Aplicaciones útiles para crear mapas conceptuales.
2. Correo electrónico.

3. Evernote, una aplicación para tomar notas en línea.

\section{CONCLUSIONES}

Los estudiantes de primer ingreso de la Facultad de Psicología de la Universidad Autónoma de Nuevo León están familiarizados con el uso de herramientas en línea para el aprendizaje. Los grupos colaborativos de Facebook y Whatsapp son la principal herramienta de aprendizaje, seguida de los portales de videos. Es importante conocer las herramientas que utilizan los estudiantes, ya que los profesores pueden incluirlas en sus cursos y facilitar el proceso de enseñanza-aprendizaje.

Para futuros estudios se recomienda aplicar esta encuesta en otras facultades para analizar similitudes 0 diferencias de acuerdo al programa de estudio. Asimismo se puede replicar el estudio con alumnos de bachillerato o posgrado para evaluar diferencias según la preparación académica.

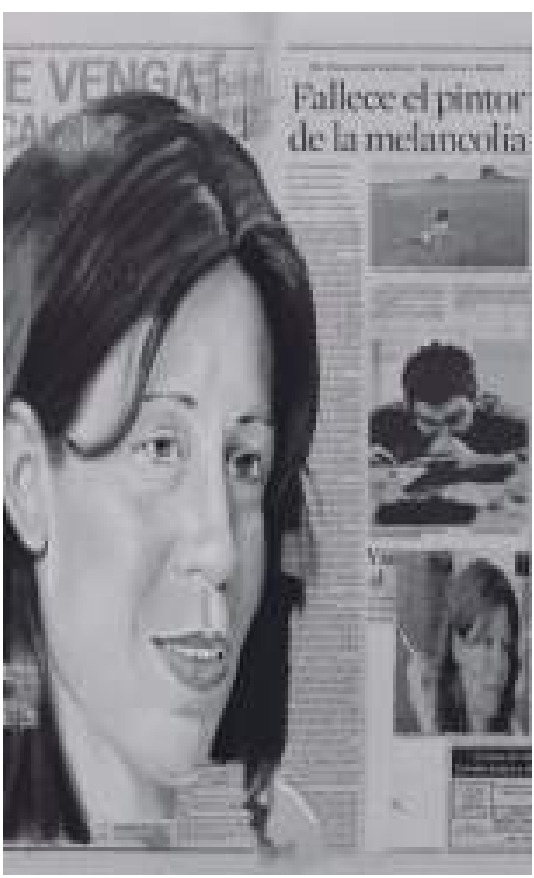




\section{REFERENCIAS}

Akamai (2016). 032016 report. Akamai's [state of the internet], 9(3), 1-68. Recuperado de: https://www.akamai.com/us/en/multimedia/documents/state-of-the-internet/q3-2016state-of-the-internet-connectivity-report.pdf

Álvarez, E., R. Ochoa, L. Salado y R. Soto (2013). La interacción de factores del modelo de videoconferencia y su influencia en el proceso de enseñanza-aprendizaje. Revista Internacional Administración \& Finanzas, 6 (4), 105-122.

Cázares, I., y A. Urbiola (2015). Estudiantes universitarios, en el uso de las herramientas tecnológicas jactivos o pasivos? Educación y Humanismo, 17(28), 15-28.

Cookson, P.y N. Domínguez (2015). Un cuento de dos países: Educación a distancia de México y Estados Unidos de América. Revista Española de Educación Comparada, 26, 61-96.

Ferro, C., A. Martínez., y M. Otero (2009). Ventajas del uso de las tics en el proceso de enseñanza-aprendizaje desde la óptica de los docentes universitarios españoles. EDUTEC, Revista Electrónica de Tecnología Educativa, 29, 1-12.

Herrera \& Batista, M. (2009). Disponibilidad, uso y apropiación de las tecnologías por estudiantes universitarios en México: perspectivas para una incorporación innovadora. Revista iberoamericana de Educación, 6(48), 1-9.

Humanante \& Ramos, P., F. García \& Peñalvo, F. y M. Conde \& González (2015). Personal Learning Environments and Online Classrooms: An experience with University Students. IEEE Revista Iberoamericana de Tecnologías del Aprendizaje, 10(1), 26-32.

Infante, A., J. Torres, J. Infante y M. Muñoz (2014). Evaluación del uso de las bases de datos electrónicas en la docencia universitaria de la Universidad de Huelva. Revista de educación a distancia, 40,1-10.

Instituto Nacional de Estadística, Geografía e Informática (2017). Estadísticas a propósito del día mundial del internet (17 de mayo). Recuperado de http://www.inegi.org.mx/saladeprensa/ aproposito/2016/internet2016__.pdf

Morales, K. (2014). ¿Cuán efectivo es el almacenamiento en la nube? Revista APEC, 30, 264 $-276$.

Navaridas, F., R. Santiago R y J. Tourón (2013). Valoraciones del profesorado del área de Fresno (California Central) sobre la influencia de la tecnología móvil en el aprendizaje de sus estudiantes. RELIEVE, 19(2), 1-20.

Prieto, J. (2013). Una aproximación metodológica al uso de redes sociales en ambientes virtuales de aprendizaje para el fortalecimiento de las competencias transversales de la universidad EAN.Virtu@lmente Revista de la Facultad de Estudios en Ambientes Virtuales, 1(1), 1-16. 


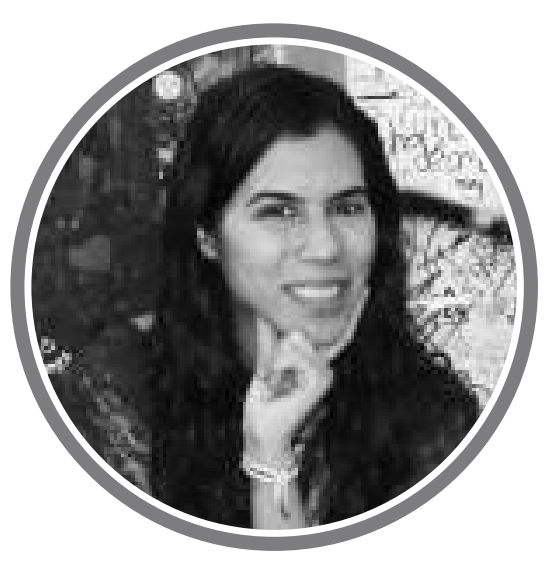

\section{Brenda Cecilia Padilla Rodríguez}

Investigadora en la Facultad de Psicología de la Universidad Autónoma de Nuevo León. Cuenta con un doctorado en Educación en Línea y Tecnologías Educativas por parte de la Universidad de Leicester en Reino Unido. Trabaja como consultora a nivel internacional, apoyando a empresas e instituciones educativas interesadas en optimizar sus procesos de aprendizaje. Su pasión es buscar formas innovadoras de diseñar cursos efectivos.

\section{Correo electrónico:}

brenda.padillardr@uanl.edu.mx

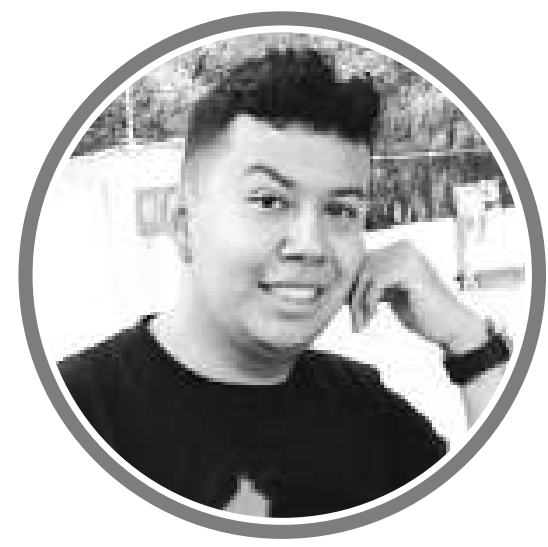

\section{Francisco Javier Rocha Estrada}

Licenciado en Psicología con acentuación Cognitivo-Conductual por parte de la Universidad Autónoma de Nuevo León, cuenta con una maestría en Ciencias con orientación en Cognición y Educación de la misma institución. Apasionado de las nuevas tendencias en educación y siempre en búsqueda de aportar nuevo conocimiento acerca del impacto del uso de la tecnología en entornos educativos.

\section{Correo electrónico:}

fcojvr25@gmail.com 